\title{
Individual working memory capacity is uniquely correlated with feature-based attention when combined with spatial attention
}

\author{
Jesse J. Bengson • George R. Mangun
}

Published online: 11 November 2010

(C) The Author(s) 2010. This article is published with open access at Springerlink.com

\begin{abstract}
A growing literature suggests that working memory and attention are closely related constructs. Both involve the selection of task-relevant information, and both are characterized by capacity limits. Furthermore, studies using a variety of methodological approaches have demonstrated convergent working memory and attention-related processing at the individual, neural and behavioral level. Given the varieties of both constructs, the specific kinds of attention and WM must be considered. We find that individuals' working memory capacity (WMC) uniquely interacts with feature-based attention when combined with spatial attention in a cuing paradigm (Posner, 1980). Our findings suggest a positive correlation between WM and feature-based attention only within the spotlight of spatial attention. This finding lends support to the controlled attention view of working memory by demonstrating that integrated feature-based expectancies are uniquely correlated with individual performance on a working memory task.
\end{abstract}

Keywords Attention · Working memory · Individual differences $\cdot$ Working memory capacity $\cdot$ Perception

Models of neural organization posit distinct pathways for location and feature template representations according to a where and what pathway (Ungerleider \& Haxby, 1994). In view of that, it is also well established that distinct neural systems may operate with respect to spatial orienting and feature attention (Ungerleider \& Haxby, 1994). Supporting evidence for such divergent cognitive mechanisms can be drawn from ERP (event-related potential) studies in which

J. J. Bengson $(\bowtie) \cdot$ G. R. Mangun

Center for Mind and Brain, University of California-Davis,

Davis, CA, USA

e-mail: jjbengson@ucdavis.edu the selection of one location in preference for another results in enhanced perceptual processing as early as $70 \mathrm{~ms}$ (Anllo-Vento, Luck, \& Hillyard, 1998). In contrast, the selection of one feature within a stimulus dimension typically induces delayed changes in perceptual processing that occur between 125-200 ms that have been localized to different neural generators (Hillyard \& Anllo-Vento, 1998). A well-represented conclusion from such studies is that feature-based attention is hierarchically dependent upon spatial attention (Mangun, 1995; Treisman \& Gelade, 1980; but see Zhang \& Luck, 2009). When viewed from the site of expression at the level of perceptual encoding, spatial and feature attention have distinct consequences; however, the domain specificity of attentional control across spatial and feature attention is less clear. Although spatial and feature attention may have distinct perceptual consequences, the top-down control of these kinds of attention may rely on a common cognitive mechanism (Fink, Dolan, Halligan, Marshall, \& Frith, 1997; Wojciulik \& Kanwisher, 1999).

Evidence for a domain general top-down control mechanism has been demonstrated through the use of functional magnetic imaging (fMRI). These studies have revealed common activation of a distributed frontal-parietal network that is at least somewhat independent of domainspecific task parameters. For example, attention to object features has been shown to pre-activate the same area of the superior parietal cortex as attention to stimulus locations (Fink et al., 1997) relative to control conditions. Furthermore, tasks that require attention to feature conjunctions (Corbetta, Shulman, Miezin, \& Petersen, 1995) and upcoming motion (Shulman et al., 1999) have also been shown to activate a proximal parietal area. Recruitment of the parietal lobe, specifically the intraparietal sulcus, has also been demonstrated across a variety of response demand conditions (Astafiev et al., 2003). Specifically, the parietal lobe was 
activated by cues that informed subjects of the anticipated manual response and anticipated location of an upcoming stimulus. These findings suggest a domain general component in for attention to space, features and action. Taken in summary, sufficient neural evidence exists to warrant a generalized attentional mechanism that operates independently of superficial task parameters (see Chiu \& Yantis, 2009 for a discussion).

This hypothesized attentional mechanism is important in the context of a discussion concerning Working Memory (WM) because a number of well-represented theoretical models propose a close link between attention and WM (Baddeley 2003; Bleckley, Durso, Crutchfield, Engle, \& Khanna, 2003; Conway, Cowan, \& Bunting, 2001; Desimone \& Duncan, 1995). Theories concerning capacity limits of WM have proposed an attentional/executive mechanism that is responsible for managing parallel domain-specific sensory processing and storage areas. Specifically, limits in the capacity of WM can be characterized as limits in the capacity of attention (Cowan, 2001; Kane, Bleckley, Conway, \& Engle, 2001). Such functional overlap has been demonstrated in behavioral studies of spatial working memory in which early perceptual processing of stimuli is improved when presented at remembered locations (Awh, Jonides, \& Reuter-Lorenz, 1998) and that when attention is deployed to a non-memorized location during a retention interval, the beneficial effects of spatial working memory are severely disrupted (Smyth, 1996). Furthermore, brain imaging studies have shown that the time course and neural locus of spatial working memory and spatial attention are so similar that spatial working memory may be best characterized as a form of retrospective spatial attention. Related studies using visual search tasks have found that search slopes increase with set size when spatial WM is occupied in a dual task scenario (Oh \& Kim, 2004; Woodman \& Luck, 2004).

When space is the domain under investigation, WM and attention seem to have a strong functional and neural overlap. However, behavioral evidence for a distinction between WM and attention has been demonstrated by Woodman, Vogel and Luck (2001a, 2001b). Woodman et al. employed a highly demanding search task during the retention interval of a visual WM task to assess the role of WM in visual search (as proposed by Bundesen, 1990) and found that visual search performance was not disrupted when visual WM was full. Such a finding suggests that visual working memory may not share a common cognitive mechanism with the form of attention required in their visual search task. In a subsequent study however, Woodman, Luck, and Schall (2008) changed the task parameters of the search task to a variable target mapping so that each trial required the generation of an internal tobe-searched-for feature template. When such trial-by-trial internal feature representations were required to perform the visual search task, the authors found that filling visual WM severely disrupted the serial deployment of attention during visual search. The trial-by-trial cueing of feature templates in the Woodman et al. study necessitated the creation and maintenance of templates requiring attention to internal feature representations called object files (Treisman \& Sato, 1990 c.f. Woodman \& Luck, 2004), which are possibly synonymous with feature-based WM representations. Thus, when viewed within the context of some of wellrepresented theories of attention, such a result suggests that visual search with variable target mapping and visual WM may share a common substrate: attention to object files. Because a visual search task is a complex cognitive process, however, this observed functional overlap between visual search and WM might also be due to the combined deployment of feature and space-based attention required of a visual search task. For example, the set-size effect observed in the task of Woodman et al. (2008) reflects a need for the serial deployment of spatial attention and eye position as well as the comparison of each possible target with the cued search template held in working memory. It may be that working memory is crucial for integrated spatial and feature-based attentional performance of this kind. Such binding is a general characteristic of attention (Treisman \& Gelade, 1980), and recent work has shown that a robust hallmark of attention (the N2PC) is specifically associated with the combined localization and identification of targets in a visual search task (Hyun, Woodman, Vogel, Hollingworth, \& Luck, 2009) but unassociated with mere target detection.

Here we test if individual variation in WMC is correlated with performance in a classic form of attention task (Posner, 1980). We further explore this effect to see if individual variation in WMC capacity predicts featurebased attention in general, combining expectancies of any sort, or if it specifically reflects integrated attentional performance across spatial and feature-based dimensions.

\section{Individual differences studies}

In the same way that neural and behavioral studies of attention have demonstrated a possible domain general capacity limit, individual differences studies have revealed a central capacity-limited mechanism responsible for WM limits that is independent of sensory-specific storage areas (Saults \& Cowan, 2007) or variation in strategic processes (Kane \& Engle, 2003). Research on individual differences has exploited the troublesome individual variability otherwise relegated to the denominator of the F-ratio to show that variation in WMC predicts performance on a number of different tasks (see Vogel \& Awh, 2008). Particularly striking is that measures of recall and recognition using complex span tasks that require the simultaneous processing and 
storage of information within high level domains such as mathematics and language predict performance on low level tasks that require attention (Kane \& Engle, 2003). One such complex span task that has been particularly fruitful is the Operation Span task (Turner \& Engle, 1989).

The OSPAN task can be thought of as a variant of a classic short-term memory task except that a secondary task is performed during the retention interval of each to-beremembered item. The consequence of such an arrangement is that rehearsal processes are disrupted, and processes that require the active maintenance of stimuli in the face of distraction are taxed. Findings from many disparate tasks and stimuli have converged to lead to the conclusion that this ability to maintain information for later recall in the face of distraction entails the ability to attend to relevant information while filtering out distracting information (Awh \& Vogel, 2008; Cowan, 2001). For example, Conway et al., (2001) tested high and low WMC participants on a version of a classic dichotic listening task (Cherry, 1953) and found that participants high in WMC were better able than low WMC subjects to filter out salient distracters in an unattended channel. Convergent evidence from a number of studies supports an attentional interpretation of WM demonstrating that individuals high in WMC are better at overcoming the reflexive orienting response induced by an exogenous cue (Kane et al., 2001), inhibiting pre-potent responses such as word naming in a Stroop task (Kane \& Engle, 2003; Long \& Prat, 2002), and have better detection accuracy for targets shown during an attentional blink time interval (Colzato, Spape, Pannebakker, \& Hommel, 2007).

Individual difference studies also provide neural evidence of attention's role in WMC using a different paradigms and stimuli. One such study is that of Vogel, McCollough and Machizawa (2005). To estimate WM, Vogel et al. (2005) employed a variant of a classic change detection task (Luck \& Vogel, 1997) in which a set of objects is presented for a short duration and then followed by either a second identical comparison array or an array in which one feature of the of the display is changed. In this task, subjects are instructed to report whether or not the second array changed. The authors modified this task to include irrelevant distracters based on either color or location, and found that a direct neural measure of distracter suppression was inversely related to WMC. Participants high in WMC were able to successfully filter out distracters, while low WMC participants unnecessarily attended to irrelevant information. When viewed within the light of evidence for a domain general attentional mechanism (Fink et al., 1997) responsible for control of access to WMC, findings such as this suggest that attention is the primary engaging factor in WM performance (Cowan, 2005).

The strong form of this attentional viewpoint is that the role of attention in working memory performance is a domain general mechanism responsible for the selection and maintenance of task relevant information in the face of distraction. Because attention is a complex cognitive component however, consideration for the specific forms of attention that interact with WM should be given. Specifically, attention usually entails both the selection of task relevant and the corresponding suppression of task irrelevant information and can also be segregated according to the perceptual domain of interest. An obvious segregation is that attention can be viewed as domain specific according to a spatial or feature-based reference. Recent work has shown that individual variability in WMC does not share any meaningful variance with the orienting response in a classic spatial cueing paradigm (Fukuda \& Vogel, 2009). When the authors included distractors at the un-cued location, however, thus combining spatial orienting with object-based distracter suppression, WMC was strongly related to performance. Related work has also shown that spatial orienting to an exogenous cue in a pro-saccade task is unrelated to variation in WMC (Kane et al., 2001). But when the authors changed the task parameters to an anti-saccade task that required the suppression of the exogenous cue and subsequent orienting to an un-cued location, variation in WMC strongly predicted performance. A related finding suggests that variation in WMC predicts the flexible allocation of spatial attention when combined with a task in which near-fixation distracters must be suppressed (Bleckley et al., 2003). When taken in summary, WM is critical for attentional performance when spatial attention is combined with other another form of attention, but plays a minimal role in the orienting response itself, whether covert or overt. The fact that WM comes into play when spatial attention is combined with distractor suppression suggests a possible role for WM in integrated attentional performance.

\section{This study}

The weight of evidence presented thus far implicates a general role of attention in WM, but given the multifaceted nature of attention (Woodman et al., 2001a, 2001b), it is important to consider what form of attention is most relevant. If an amodal attentional mechanism exists (Esterman, Chiu, Tamber-Rosenau, \& Yantis, 2009; Fink et al., 1997; Wojciulik \& Kanwisher, 1999) that is responsible for WM performance (Cowan, 2005, 2001), such domain-specific parameters should not be relevant. If it is true that a generalized attention system exists that drives WM performance, then loading visual WM should also interfere with performance on a visual search task with consistent target mappings. This is not the case, however (Woodman et al., 2001a, 2001b). In a similar fashion, if WMC, as measured by the OSPAN task, reflects variation in 
a general attentional control mechanism, it should also vary with performance on a similar version of a visual search task. This is not the case (Kane, Poole, Tuholski, \& Engle, 2006). Instead, when taken in summary, the evidence presented thus far suggests specific boundary conditions for the role of attention in WM.

The present study refines the theoretical fidelity of attention's role in individual's WMC by testing the relationship of individual WMC to feature-based, response-related, and spatial attention on a within-subjects basis. We employ a combined expectancy paradigm (Handy, Green, Klein, \& Mangun, 2001; Kingstone, 1992). The combined expectancy paradigm used here is a variant of a classic Posner cuing paradigm (Posner, 1980) in which subjects are validly or invalidly cued to the spatial and feature characteristics of upcoming stimuli. We present a series of four experiments to show that individual variation WMC predicts performance in a variant of a classic expectancy task, but only when this task necessitates the binding of feature and spatial attention. We replicate this finding to show that this correlation is not a function of general task difficulty (Experiment 2). We then show that it is unrelated to response cuing or simply the binding of any two types of cues (Experiment 3), and unrelated to feature-based attention presented in isolation at fixation without any spatial cueing (Experiment 4). The demonstrated correlation of individuals' WMC with prospective attention lends support to the controlled attention view of WM, and the specificity with which this correlation occurs suggests a role for WM in integrated feature-based attentional performance.

\section{Experiment 1}

Methods

\section{Participants}

Twenty-eight University of California-Davis undergraduates participated for research credit as a requirement for completion of an introductory psychology class. All had normal or corrected-to-normal vision. All correct trials were entered into statistical analysis, and data from all participants were included in subsequent analyses.

\section{Expectancy task}

Apparatus

Stimuli were presented on a 19' Viewsonic VX922 color monitor placed $60 \mathrm{~cm}$ from each participant's nasion.
Stimuli

Each trial began with the $200 \mathrm{~ms}$ presentation of an arrow $\left(1.8^{\circ}\right.$ long $)$ at fixation with the letter $\mathrm{V}$ or $\mathrm{H}\left(.7^{\circ} \times .9^{\circ}\right)$ positioned directly above it. Following a randomly distributed Stimulus-Onset Asynchrony of $700 \pm 100 \mathrm{~ms}$, vertically or horizontally aligned $5^{\circ}$ square target gratings were presented for $100 \mathrm{~ms}$ at location markers $11.5^{\circ}$ to the right or left of a white dot placed at fixation and $3.5^{\circ}$ below the horizontal meridian at a proportion of .50 . Spatial frequency of gratings subtly varied randomly and evenly within each condition and hemi-field at a proportion of .50 between high $\left(.53^{\circ}\right.$ per cycle) and low $\left(.59^{\circ}\right.$ per cycle) spatial frequencies of alternating white and black square waves. Stimuli were presented on a gray background (rgb intensities of $60,60,60$ ) with an inter-trial interval of 2,000 ms. Each trial proceeded automatically. Figure 1 illustrates an exemplar trial sequence and target gratings, and Fig. 2 illustrates the subtle response-relevant difference between high and low spatial frequencies of gratings.

\section{Procedure}

Participants were instructed to make a 2-AFC discrimination between the high and low spatial frequency dimensions of the target gratings. Participants were also instructed to maintain gaze at fixation point for the duration of each trial block. A dummy electrode was also placed on the left temple, and participants were told that horizontal eye movements were being monitored using the electrode. Eye movements were also monitored by the experimenter using a high-resolution camera. Critically, participants were instructed to use the cue to anticipate the orientation and location of the impending target grating and to respond as quickly and accurately as possible to the spatial frequency of the gratings.

Trials were administered in 16 4-min blocks of 60 trials each. Each block contained 48 trials in which both orientation and location were validly cued: 4 trials with only validly cued orientation, 4 trials with only validly cued spatial location, and 4 trials with both dimensions invalidly cued. This resulted in within-subjects $2 \times 2$ spatial by

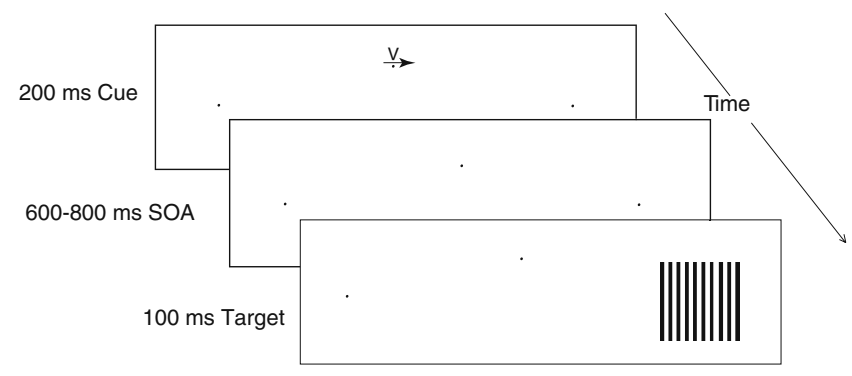

Fig. 1 Illustrates an exemplar trial sequence 


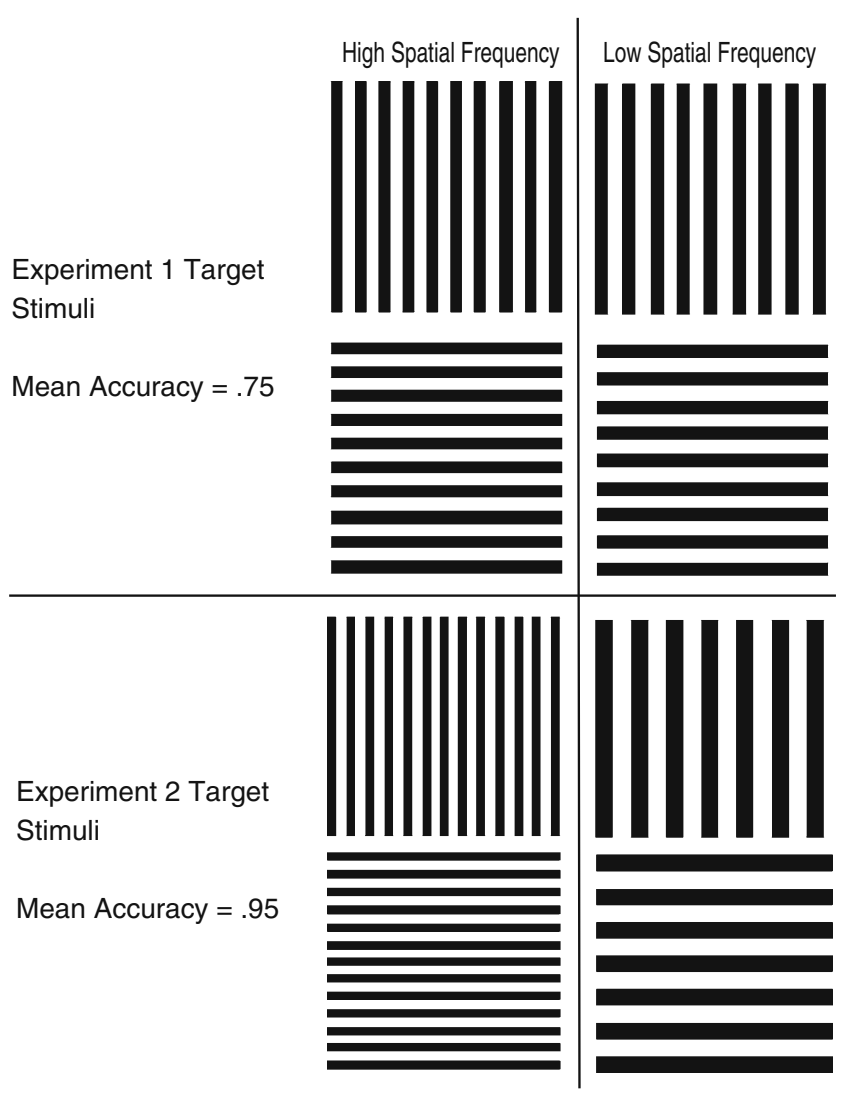

Fig. 2 Illustrates the subtle response-relevant difference between high and low spatial frequencies of gratings

orientation cueing factorial design where each cue was .867 predictive of its particular dimension. Targets appeared with equal probability (.50) in the left and right hemi-field and orientation of targets varied randomly with a proportion of .50 within each cueing condition and spatial hemi-field. Trial order was also randomized using a Mersene twister algorithm.

\section{OSPAN task}

Counterbalanced to administration prior to or after the expectancy task, working memory capacity was measured using a classic version of the Operation Span task (Kane \& Engle, 2003; Turner \& Engle, 1989). Participants were presented with an operation string such as " $4 / 2+2=9$ ? L", and were instructed to read the equation and answer out loud, report whether the given answer is correct or incorrect, and then read the letter out loud. The importance of correctly answering math questions was emphasized, and subjects were instructed to "do your best" when recalling letters. Progression from trial to trial was experimenter controlled with a mouse click, and 12 series of 2 to 5 serially presented trials were administered. At some point in each series (depending on set size: 2-5), participants were visually cued to recall at which point they attempted to remember and write down the letters in their order of presentation. All series in which a participant correctly recalled all letters in the correct order were summed to produce OSPAN scores ranging from 0-42. In experiment 2, as a supplementary demonstration of external validity, we used the subject-administered automated version of the OSPAN task in which subjects perform a recognition instead of recall task at test (Unsworth, Heitz, Schrock \& Engle 2005).

\section{Experiment 1 results}

Reaction times

Table 1 contains mean reaction times (RTs) in milliseconds and proportion correct (ACC) for each condition for high and low span groups with significant simple effects marked with an asterisk. Mean RTs for each of the four conditions were entered into a repeated measures $2 \times 2$ ANOVA with location (expected vs. unexpected) and orientation (expected vs. unexpected) as within-subjects categorical factors. Analysis of individual differences was conducted with OSPAN entered into the model as a between-subjects continuous predictor (Cohen, Cohen, West, \& Aiken, 2003; Hutchison, 2007). Results indicate a significant 3-way orientation $\times$ spatial $\times$ OSPAN interaction, $\mathrm{F}(1,26)=$ $6.097, p=.02, \eta^{2}=.190$, demonstrating that OSPAN covaried specifically with orientation cuing, but only at validly cued spatial locations. Contrast analysis of main effects revealed no significant effect of orientation validity over all subjects collapsed over spatial validity, $\mathrm{F}(1,26)=.0$, $p=.994, \eta^{2}=.000$, nor did the effect of orientation validity interact with OSPAN performance when collapsed across spatial validity, $\mathrm{F}(1,26)=.133, p=.718, \eta^{2}=.005$. In contrast, a significant spatial cuing main effect was observed when data were collapsed across orientation validity, $\mathrm{F}(1$, 26) $=7.053, p=.013, \eta^{2}=.213$. This spatial cuing effect did not significantly vary as a function of orientation validity $(p=.196)$ and did not interact with OSPAN performance $(p=.707)$. For further illustrative purposes, the bottom right of Fig. 3 plots the correlation of OSPAN performance with the orientation cuing effect at validly cued spatial locations. It is also important to note that the three-way interaction reported above and the subsequent analyses point to the specificity with which an association between OSPAN and orientation cuing is observed. Only at validly cued spatial locations was a significant orientation cuing effect demonstrated, and it was this effect that was significantly related to OSPAN performance $(r=.387, p=.042)$. Orientation cuing at invalidly cued spatial locations was near zero over all subjects and did not correlate with OSPAN performance $(r=-.217$, $p=.267)$. Such a finding speaks to the hierarchical dependence of feature expectancies on spatial attention 
Table 1 Experiment 1. [For all tables, reaction time (RT) is in milliseconds and Accuracy (ACC) is proportion correct]
Valid location

RT (ACC)

$655(.79)$

$673(.82)$

$18(-.03)^{*}$

$657(.82)$

$706(.84)$

$49 *(-.02)$

Orientation cuing

Low OSPAN

Valid orientation

Invalid orientation

Orientation cuing
Invalid location

Spatial cuing RT (ACC)

$\begin{array}{llll}\text { All subjects } & & & \\ \text { Valid orientation } & 655(.79) & 764(.71) & 109 *(.08)^{*} \\ \text { Invalid orientation } & 673(.82) & 757(.68) & 84^{*}(.14)^{*} \\ \text { Orientation cuing } & 18(-.03)^{*} & -7(.03)^{*} & \\ \text { High OSPAN } & & & 142^{*}(.09)^{*} \\ \text { Valid orientation } & 657(.82) & 799(.73) & 81^{*}(.12)^{*} \\ \text { Invalid orientation } & 706(.84) & -12(.02) & \\ \text { Orientation cuing } & 49 *(-.02) & 708(.69) & 67 *(.06)^{*} \\ \text { Low OSPAN } & & 726(.65) & 84^{*}(.14)^{*} \\ \text { Valid orientation } & 641(.75) & 18(.04) & \\ \text { Invalid orientation } & 642(.79) & & \\ \text { Orientation cuing } & 1(-.04) & & \end{array}$

(Mangun, 1995) and that this effect is specifically related to OSPAN performance.

For generalizability to other common methods of analysis (Hutchison, 2007) using OSPAN, and for demonstrative purposes, we also conducted a tertiary split (Fig. 3) and analyzed group differences as a function of high, middle, and low OSPAN groups. Data from each span group were entered into a $2 \times 2$ repeated measures ANOVA. Analysis of high spans (OSPAN $>20, N=9$ ) revealed a significant spatial by feature cueing interaction, $\mathrm{F}(1,8)=13.251, p=$
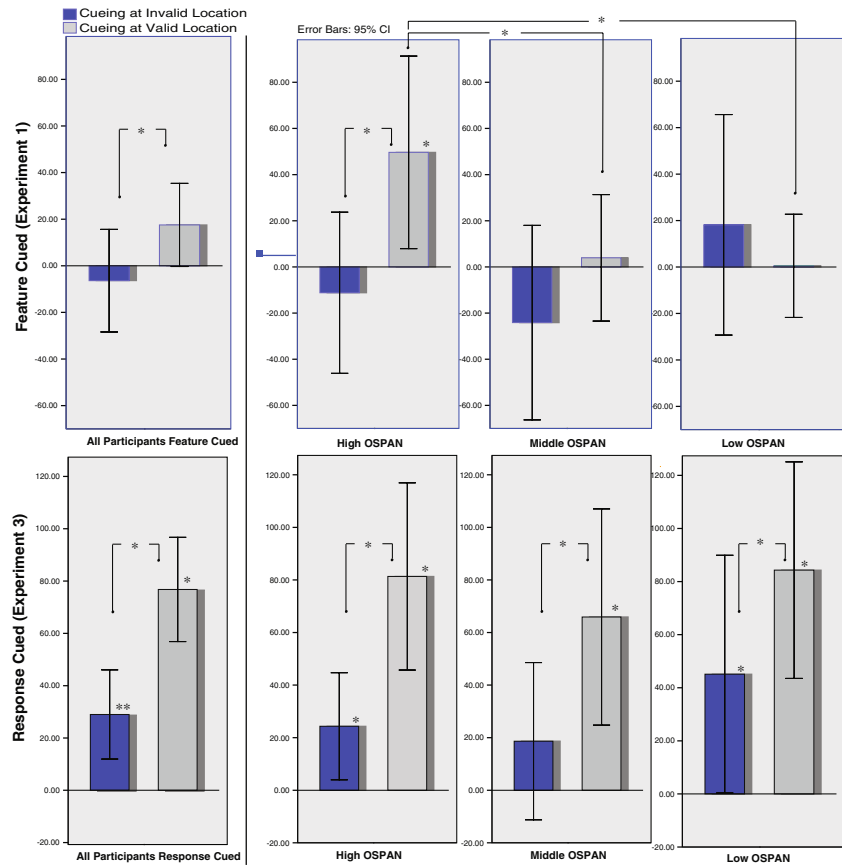

Fig. 3 Plots effect of feature attention on the y-axis (invalid-valid orientation reaction times) at validly cued (light bars) and invalidly cued (dark bars) locations indicating a focal feature expectancy effect centered at validly cued locations only for participants high in working memory capacity. A more distributed effect across subjects and spatial locations is observed when responses are cued [Experiment
$.007, \eta^{2}=.624$. Further analysis of simple effects revealed a significant effect of orientation validity at validly cued spatial locations, $\mathrm{t}(8)=2.744, p=.025$, but not at invalidly cued spatial locations, $\mathrm{t}(8)=-.739, p=.481$. In contrast, the $2 \times 2$ interactions for middle, $\mathrm{F}(1,8)=1.856, p=.206, \eta^{2}=$ .171 , and low $\mathrm{F}(1,8)=.523, p=.490, \eta^{2}=.061$, spans were not significant. Furthermore, analysis of simple effects revealed no orientation cueing for either middle $(p=.417)$ or low spans $(p=.398)$ at validly cued spatial locations. In fact, high spans exhibited a significantly greater orientation

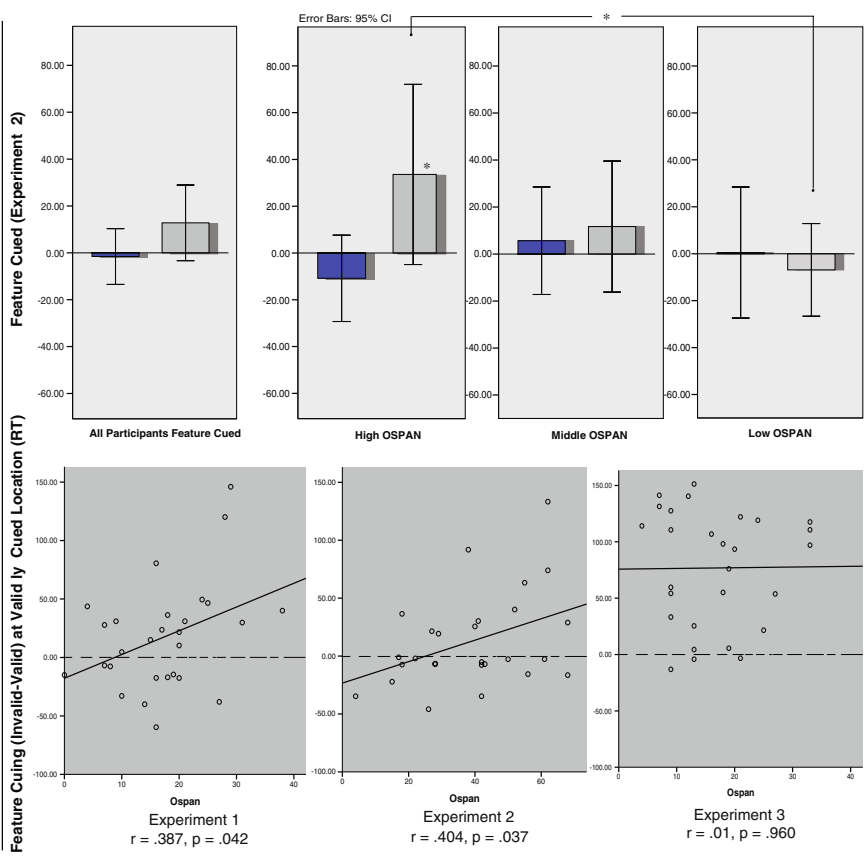

3 (bottom left)]. All significant or marginally significant within- and between-subject interactions are displayed (both valid and invalid spatial locations) with asterisks. Bottom right of Figure 3 depicts correlations between orientation cuing (invalid-valid reaction times for validly cued locations) and individual WMC across all subjects for Experiments 1-3 

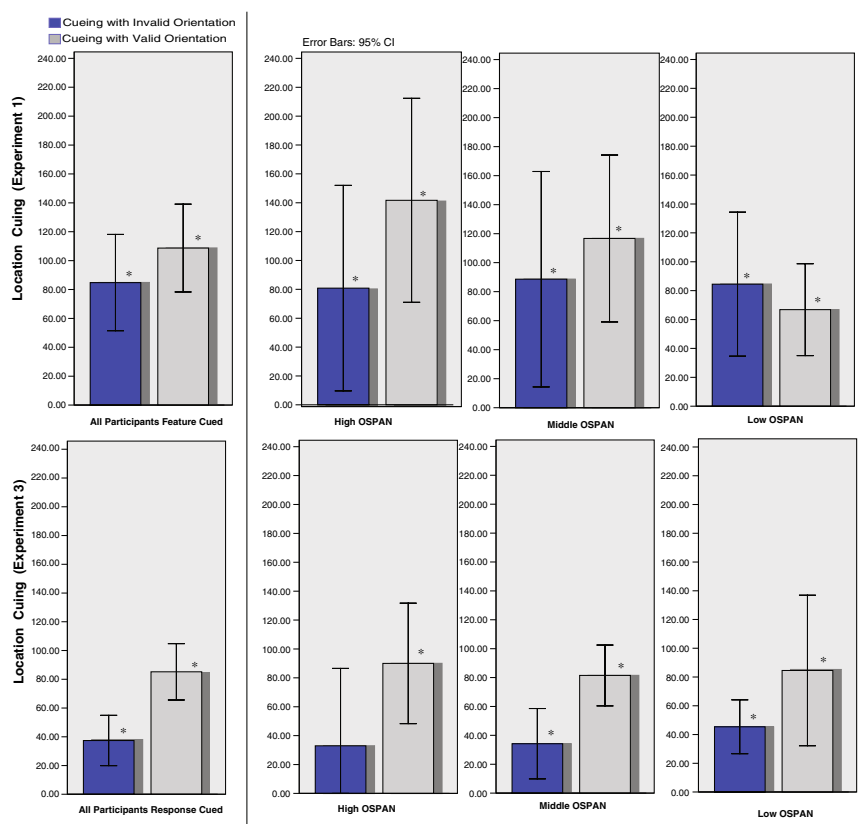

Fig. 4 Illustrates effect of spatial attention on the y-axis (invalid-valid location reaction times) for validly cued (light bars) and invalidly cued orienations (dark bars) on the x-axis indicating a distributed spatial expectancy effect for both validly and invalidly cued orientations for all span-groups across Experiments 1-3. All signifi-

cuing effect than both middle and low spans. Repeated measures ANOVA with OSPAN as a between-subjects categorical factor reveal significantly greater orientation cuing at validly cued spatial locations for high vs. low spans, $\mathrm{F}(1,16)=5.986, p=.029, \eta^{2}=.264$ and high vs. middle spans, $\mathrm{F}(1,17)=6.285, p=.047, \eta^{2}=.212$.

In contrast to the specificity with which feature cuing effects occurred, Fig. 4 illustrates significant effects of location validity irrespective of the validity of the orientation cue or of span group. Whereas only those subjects in the top third of OSPAN performance showed significant feature cuing only at validly cued spatial locations, all three subject groups demonstrated significant (all $p \mathrm{~s}<.05$ ) location cuing effects irrespective of the validity of the orientation cue. Furthermore, the correlation of OSPAN with spatial cueing was non-significant (see bottom right of Fig. 4), in contrast with the significant correlation between OSPAN and orientation cueing shown in Fig. 3.

A null correlation is prone to a number of factors, however, such as low reliability or restriction of intersubject variability. To address these issues, we examined the split-half reliability of the spatial and feature-based cuing effects using the Spearman-Brown prophecy correction (Brown, 1910; Spearman, 1910) ${ }^{1}$. For Experiment 1

\footnotetext{
${ }^{1}$ We would like to thank Dr. Keith Widaman for his help with this analysis.
}

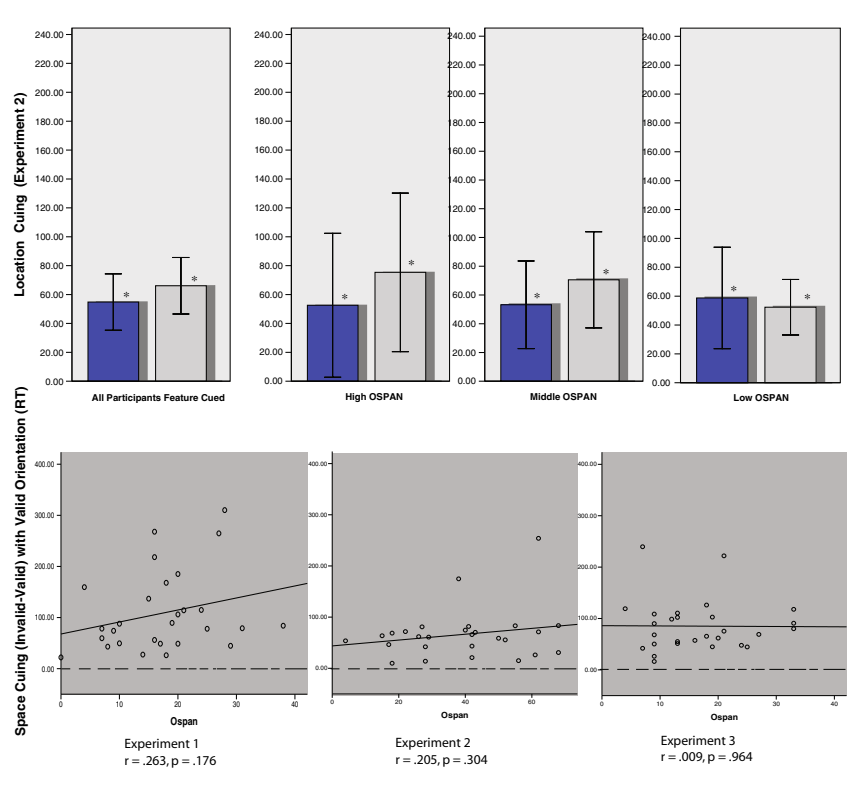

cant $(p<.05)$ spatial cueing effects are displayed with asterisks. Bottom right of figure depicts correlation of spatial attention (invalidvalid reaction times for validly cued orientations) across all subjects for Experiments 1-3

the split-half reliability for the spatial cuing effect was $r=$ .77 while the reliability for the orientation cuing effect was comparable at $r=.73$, suggesting that insufficient reliability for the spatial cueing measure is not the critical factor. The second related issue concerns the range of each respective cuing effect. All span groups demonstrated significant spatial cuing, and the lack of correlation with WMC may be due to a restriction of range across subjects (not enough between subject variability). When we examine the range of the spatial cuing effect across subjects for Experiment 1, however, we observe that the range is $288 \mathrm{~ms}$ (minimum = $22 \mathrm{~ms}$; maximum $=310 \mathrm{~ms}$ ) while the range for the orientation cuing effect is actually more restricted at $205 \mathrm{~ms}$ $($ minimum $=-59 \mathrm{~ms} ;$ maximum $=146 \mathrm{~ms})$.

\section{Accuracy}

Mean proportion correct for each of the four withinsubjects conditions were also entered into a repeated measures ANOVA with OSPAN as a covariate. Results revealed no significant three-way interaction, $\mathrm{F}(1,26)=$ $1.789, p=.193, \eta^{2}=.064$. Analysis of main effects revealed no significant effect of orientation validity, $\mathrm{F}(1,26)=.178, p=.676, \eta^{2}=.007$ collapsed across spatial validity, but a main effect of spatial validity, $\mathrm{F}(1,26)=10.791, p=.003, \eta^{2}=.293$. Neither effect interacted with OSPAN performance $(p s>.60)$. In 
summary, only spatial validity significantly increased accuracy, and neither orientation nor spatial validity interacted with OSPAN performance $(p \mathrm{~s}>.50)$.

\section{Discussion}

Results of Experiment 1 suggest that variation in WMC is strongly associated with feature-based attention only within the spotlight of spatial attention. Importantly, divergent methods of analysis using OSPAN as either an extreme group or continuous measure of individual variability converge on the same conclusion: WMC is critically associated with integrated feature-based attention when combined with spatial attention.

The literature presents a case for an attentional view of WM; however, no study has explored the common type of prospective attention required of an expectancy paradigm (Posner, 1980). Here we provide evidence that WMC covaries with feature-based attention when combined with spatial attention in an integrated cuing paradigm. The results of Experiment 1 lend support to the controlled attention view of WM demonstrating that variation in WMC is related to the prospective deployment of featurebased attention when combined with spatial attention.

\section{Experiment 2}

Given the small sample size and the novelty of the results in Experiment 1, a replication of this pattern is warranted and may serve as the best statistic (Falk, 1998). Such a demonstration is important for a number of reasons, the first of which is that, with respect to the significant association of OSPAN with feature cuing within the spotlight of attention, we may have simply been lucky in our sample selection.

Another reason stems from an effort to achieve a degree of generalizability (Fisher, 1966). Experiment 1 used target stimuli under conditions of high perceptual load. That is, the discrimination task at target presentation was quite difficult as estimated by an average subject accuracy of $75 \%$ correct. Previous studies have demonstrated that increases in perceptual load lead to a greater taxing of resource-limited attentional mechanisms, whereby significantly greater behavioral (Lavie \& Tsal, 1994) and perceptual consequences (Barnhardt, Ritter, \& Gomes, 2008; Handy \& Mangun, 2000) of attention are observed under conditions of greater target processing difficulty. Thus, if WM and attention share a common capacitylimited resource, the demonstrated significant association between individual's WMC and attention may be specific to conditions of difficult target discrimination. However, if task difficulty is decreased and the pattern of results found in Experiment 1 replicates, then we can more confidently claim that variation in WMC is generally related to prospective attention and not to an interaction between task difficulty and WMC.

\section{Methods}

Participants

Twenty-eight University of California-Davis undergraduates participated for research credit as a requirement for completion of an introductory psychology class. All had normal or corrected-to-normal vision. All trials were entered into statistical analysis, and data from one participant were removed due to failure to maintain accuracy of $85 \%$ on the mathematical part of the OSPAN task.

Methods and stimuli were identical to Experiment 1 with two exceptions. The first is that the difference in spatial frequency was made much greater, thus decreasing task difficulty at target presentation. Experiment 1 used gratings that varied in spatial frequency between $.53^{\circ}$ and $.59^{\circ}$ cycles per degree. In experiment 2 , we use gratings that varied between spatial frequencies of either $.38^{\circ}$ or $.71^{\circ}$ cycles per degree. A manipulation check revealed that discrimination accuracy increased significantly from $75 \%$ over all subjects in Experiment 1 to $95 \%$ in Experiment 2. The other difference is that, in Experiment 2, we employed an automated version (Unsworth et al., 2005) of the operation span task that uses a serial recognition task as the dependent measure rather than a serial recall task. This was done for two reasons: to provide a demonstration of the external validity of our individual differences measure, and because it is much easier to administer and score the automated version than the classic version. The automated OSPAN is a mousedriven program that allows the measure of Working Memory Capacity independently of the experimenter. Trials consisted of set sizes of 3-7 with a total of 75 letters and math problems. Memory for the letters was tested using a $4 \times 3$ matrix of letters, and subjects used a mouse to check which letters were presented at encoding in the order of presentation. Feedback concerning accuracy for the mathematical portion of the task was also given in red numbers in the upper right-hand corner of the screen, and subjects were encouraged to maintain an accuracy of at least $85 \%$. Scores on this task could range between $0-75$.

\section{Results}

Reaction Times

Table 2 contains mean reaction times (RTs) in milliseconds and proportion correct for each condition for high and low 
Table 2 Experiment 2

\begin{tabular}{llll}
\hline & $\begin{array}{l}\text { Valid location } \\
\text { RT (ACC) }\end{array}$ & $\begin{array}{l}\text { Invalid location } \\
\text { RT (ACC) }\end{array}$ & $\begin{array}{l}\text { Spatial cuing } \\
\text { RT (ACC)__ }\end{array}$ \\
\hline $\begin{array}{l}\text { All subjects } \\
\text { Valid orientation }\end{array}$ & $538(.96)$ & $604(.95)$ & $66^{*}(.01)^{*}$ \\
Invalid orientation & $551(.95)$ & $606(.95)$ & $55^{*}(.00)$ \\
Orientation cuing & $13(.01)$ & $2(.00)$ & \\
High OSPAN & & & $76^{*}(.01)$ \\
Valid orientation & $571(.96)$ & $647(.95)$ & $53^{*}(.02)$ \\
Invalid orientation & $605(.96)$ & $658(.94)$ & \\
Orientation cuing & $34(.00)$ & $11(.01)$ & $52^{*}(.01)$ \\
Low OSPAN & & $590(.94)$ & $59^{*}(.01)$ \\
Valid orientation & $538(.95)$ & $590(.94)$ & \\
Invalid orientation & $531(.95)$ & $0(.00)$ & \\
Orientation cuing & $7(.00)$ & &
\end{tabular}

span groups with significant simple effects marked with an asterisk. As with Experiment 1, mean RTs for each condition were entered into a repeated measures ANOVA with OSPAN as a covariate. Replicating Experiment 1 almost exactly, results indicate a significant three-way orientation $\times$ spatial $\times$ OSPAN interaction, $\mathrm{F}(1,25)=5.261, p=.03$, $\eta^{2}=.174$, demonstrating that orientation cuing depended upon validly cued locations and individuals' WMC. Again, analysis of main effects revealed no main effect of orientation validity, $\mathrm{F}(1,25)=.693, p=.413, \eta^{2}=.027$, nor did this main effect interact with OSPAN, $\mathrm{F}(1,25)=2.162, p=.154, \eta^{2}=$ .08. However, replicating Experiment 1, a significant main effect of location validity was observed irrespective of OSPAN or orientation validity, $\mathrm{F}(1,25)=6.405, p=.018$, $\eta^{2}=.204$, that did not interact with OSPAN, $\mathrm{F}(1,25)=.063$, $p=.804, \eta^{2}=.002$. Figure 3 plots the correlation of OSPAN scores with orientation cuing at validly cued spatial locations. In replication of Experiment 1, significant orientation cuing was not found at invalidly cued spatial locations, and orientation cuing at invalidly cued spatial locations did not correlate with OSPAN performance $(r=-.024, p=.904)$. Also, in replication of Experiment 1, the bottom right of Fig. 4 illustrates no significant correlation of OSPAN with spatial cuing $(r=.205, p=.304)$.

Although not as striking as Experiment 1, analysis of group differences according to a tertiary split revealed a general replication (Fig. 3, top right) of the pattern found in Experiment 1. Analysis of high spans (OSPAN $>50, N=9$ ) revealed a trend towards a spatial by feature cueing interaction, $\mathrm{F}(1,8)=$ $3.137, p=.114, \eta^{2}=.282$, with a marginally significant orientation cuing effect at validly cued spatial locations, $\mathrm{F}(1,8)=4.039, p=.079, \eta^{2}=.336$, but not at invalidly cued locations, $\mathrm{F}(1,8)=1.827, p=.213, \eta^{2}=.186$. The $2 \times 2$ interaction for middle spans, $\mathrm{F}(1,8)=6.137, p=.036, \eta^{2}=$ .441 , was also significant, but was not significant for low spans, $\mathrm{F}(1,8)=.170, p=.691, \eta^{2}=.021$. Analysis of simple effects with orientation cuing at validly cued spatial locations revealed no between subjects interaction between high and middle spans for orientation cuing, $\mathrm{F}(1,16)=1.125, p=.305$, $\eta^{2}=.066$. Importantly, however, there was significantly higher orientation cuing for high spans compared with low spans, $\mathrm{F}(1,16)=4.649, p=.047, \eta^{2}=.225$. It is important to note that this high vs. low comparison is most typical of extreme groups designs using OSPAN and is in proximate replication of Experiment 1's high vs. low comparison.

As in Experiment 1, Fig. 4 illustrates that all three span groups demonstrated significant location cueing effects (all $p$ s < .05). Furthermore, no span group exhibited significant between-group differences in spatial cuing from any other span group (all $p \mathrm{~s}>.30$ ). The reliability for the spatial cuing effect was $r=.83$ and $r=.63$ for the orientation cuing effect. Experiment 2 also produced an inter-subject range of $244 \mathrm{~ms}$ (minimum $=10 \mathrm{~ms}$; maximum $=253 \mathrm{~ms})$ for the spatial cuing effect and $179 \mathrm{~ms}$ (minimum $=-46$; maximum $=$ $133 \mathrm{~ms}$ ) for the orientation cuing effect. Thus, insufficient reliability and insufficient inter-subject variability do not seem to be the determining factor in our observed insignificant correlation between WMC and spatial orienting.

\section{Accuracy}

Results of a repeated measures ANOVA with OSPAN as a covariate revealed no significant three-way interaction when proportion correct was the dependent variable, $\mathrm{F}(1,25)=.801, p=.379, \eta^{2}=.031$. Analysis of main effects revealed no effect of orientation, $\mathrm{F}(1,25)=.016$, $p=.902, \eta^{2}=.001$, or of spatial validity, $\mathrm{F}(1,25)=1.557$, $p=.224, \eta^{2}=.059$. In summary, neither location nor orientation cue validity affected accuracy, nor did either effect interact with OSPAN performance $(p s>.30)$. 


\section{Discussion}

The pattern of results found in Experiment 2 replicates the pattern found in Experiment 1 suggesting that prospective feature attention is associated with variation in WMC within the spotlight of spatial attention. Particularly striking is that the overall correlation of OSPAN scores with orientation cuing is almost identical across Experiments 1 and 2. Thus, under conditions of decreased task difficulty and with different measures of WMC, the results of Experiment 2 indicate that prospective feature-based attention in a combined expectancy task is significantly related to individual variability in WMC. Furthermore, Experiment 2 replicates the null association found in Experiment 1 between spatial attention and WMC. A qualitative interpretation of such a result is that feature-based attention is unique in its relationship to variation in WMC and that spatial attention operates at least somewhat independently. This qualitative interpretation is not necessarily an inescapable conclusion however. A single domain general resource account might still be employed to explain our pattern of findings. This explanation stems from a fundamental proposition: all forms of attention, whether prospective (cuing) or retrospective (WM), rely on a single resource limited mechanism. This resource-based quantitative account states that spatial attention merely does not tax this domain general mechanism sufficiently and that high spans actually excel in the coordinated deployment of prospective attention because they have leftover attentional capacity with which to use the feature-based cues. Thus, rather than being a qualitative distinction, this quantitative account suggests that high spans excel under conditions that require more attentional resources. Thus, the additional deployment of feature-based attention combined with spatial attention is why variation in WMC comes into play. To be sure, given the hierarchical nature of our effect, that feature-based attention relies on spatial attention, such an account has possible merit. If such a domain general attentional resource exists, then it is reasonable to propose that the shared variance between feature-based attention and WMC should also be at least partly shared with spatial attention. In fact, there exists a positive, although nonsignificant trend in Experiments 1 and 2 between spatial cuing effects and individuals' WMC. It may be that that this shared variance between WMC and spatial attention is also shared with feature-based attentional performance. Conversely, the observed co-variation between feature cuing and WMC might be statistically independent of the correlation between spatial attention and WMC as the qualitative account would predict. Our analyses thus far do not explore this possibility and the low number of subjects in Experiments 1 and 2 might not have enough power to answer this question. To address this issue more thoroughly, we first normalized the OSPAN scores for Experiments 1 and 2 in order to have a common dispersion (since two different versions of the task were used that produced arbitrarily different ranges) and then entered the cuing effects into a $2 \times 2$ within-subjects ANOVA with Ospan (high vs. middle vs. low tertile split) and Experiment (1 vs. 2) as an additional between-groups factor. The results of this ANOVA revealed no significant location $\times$ orientation cueing $\times$ OSPAN $\times$ Experiment $(1$ vs. 2$)$ interaction, $\mathrm{F}(2,49)=1.006$, $p=.373, \eta^{2}=.039$. Thus, the relationship of WMC to attentional performance did not vary as a function of experiment. As a result, all subsequent analyses were collapsed across Experiments 1 and $2(N=55)$. The results of a $2 \times 2$ within-subjects ANOVA with OSPAN as a covariate collapsed across Experiments 1 and 2 reveal a robust location $\times$ orientation $\times$ OSPAN interaction, $\mathrm{F}(1,53)=$ $10.670, p=.002, \eta^{2}=.168$. As in each of Experiments 1 and 2 , individual OSPAN scores interacted with the feature cuing effect only at the validly cued location, $\mathrm{r}=.393, p=.003$. No other correlations were significant (all $p$ values $>.10$ ); however, the correlation of WMC with spatial cueing produced a positive trend, $r=.224, p=.101$, suggesting possible shared variance among feature cueing, spatial attention, and WMC. To explore whether these two correlations were driven by common variance, we entered the spatial cuing scores for each subject as the first predictor in a hierarchical linear regression model with the orientation cuing effect added as an additional independent predictor of OSPAN scores. This procedure offers a test of whether feature-based attention shares independent variance with WMC after having accounted for the possible shared variance between spatial attention and WMC. The results reveal that after having accounted for this shared variance, feature-based attention still robustly predicts variation in WMC, $\beta=.363, t=2.83, p=.007$, independent of any shared variation with spatial attention. In fact, after having accounted for the effect of spatial attention, the shared variance between WMC and feature-based attention is still $12.7 \%\left(r=.356^{2}\right)$. Without spatial cuing effects in the model, the percentage of variance shared between the orientation cuing effect and WMC was $15.5 \%\left(.393^{2}\right)$. This suggests that the association between WMC and feature-based attention is almost completely unique in its shared variation with individuals' WMC. If a domain general resource-limited attentional mechanism is driving our results, we would not expect such a unique correlation. Conversely, the amount of independent variance shared between spatial attention and WMC is $2.2 \%$ of a possible $5.0 \%$ total amount possible $\left(r=.224^{2}\right)$.

There remain differences in the symbolic nature of the cues themselves however. The spatial cue used an arrow that more directly maps onto the associated expectancy, whereas the letter cue for orientation requires a symbolic transformation into its associated instructional meaning ("V" for vertical and " $\mathrm{H}$ " for horizontal). In everyday 
life, arrows provide instructional commands in and of themselves, whereas the meaning of either a "V" or "H" in this study requires a symbolic transformation of each letter into its anticipated feature representation in specific terms of the task instructions. Thus, even though the cues were predictive most of the time, the correlation between OSPAN and feature-based attention may have been due to the greater efficiency with which the high spans can keep task instructions (Kane \& Engle, 2003) in mind. A second, but related issue is that our measure of WMC used letters as a dependent measure. Thus, the specific overlap in performance between feature attention and WMC demonstrated here may be a function of the orthographic nature of the feature cue itself. A third issue is that WMC might reflect performance of any combined attention task. The combined cuing paradigm we employ here requires the coordination of two distinct cues to anticipate the location and orientation of an impending target. A possible interpretation of the results of Experiments 1 and 2 is that high spans excel in the use of two separate sources of information to integrate expectancies and prepare for the upcoming target. The question of whether this coordination of attention generalizes to other attentional domains remains to be resolved however. If WMC is related to the integration of combined expectancies in general, then it should also predict performance when the letter cue provides information concerning the impending correct response (as in Astafiev et al., 2003). However, if WMC is specific to feature-based attention, we would expect no correlation with performance under these task parameters. By cueing responses in this way, an expectancy for the orientation of the impending target can instead be relegated to a motor expectancy with no requisite for the generation of an anticipated internal feature-based template bound to a location in space. If WMC is especially critical for feature-based attentional performance, then instructing subjects to respond to the target orientation should remove the role of WMC in this combined cuing paradigm.

\section{Experiment 3}

Method

\section{Participants}

Twenty-eight University of California-Davis undergraduates participated for research credit as a requirement for completion of an introductory psychology class. All had normal or corrected-to-normal vision. All trials were entered into statistical analysis, and data from all participants were included in subsequent analyses.
Experiment 3 was identical to Experiment 1 except that one sentence of the instructions was changed. Instead of "respond to the spatial frequency of the target gratings," subjects were instructed to "respond to the orientation of the gratings." In this way, cue and response preparations were perfectly confounded. Such a manipulation allows for control of the differential interpretive and instructive difficulty of the orthographic vs. arrow cues. A failure to replicate the interaction between OSPAN and feature attention would suggest that differences in the predictive utility of OSPAN is not driven by differences in the natures of the cues themselves. In both Experiments 1 and 3 subjects would have to keep task instructions in mind that direct the symbolic transfer of the orthographic cue into its associated task-specific instructional representation. The difference in Experiment 3, however, is that the letter cue provides information concerning the anticipated correct response, thus bypassing the need to generate an internal feature representation bound to an upcoming cued location that is required for the observed orientation cuing effects in Experiments 1 and 2.

\section{Results}

\section{Reaction Times}

Table 3 contains mean reaction times (RTs) and proportion correct for each condition for high and low span groups with significant simple effects marked with an asterisk. Results of a repeated measures ANOVA reveal no significant location $\times$ response cueing $\times$ OSPAN interaction, $\mathrm{F}(1,26)=1.683$, $p=.206, \eta^{2}=.061$. In contrast to Experiments 1 and 2, analysis of main effects revealed a significant main effect of response validity, $\mathrm{F}(1,26)=11.636, p=.002, \eta^{2}=.309$. This main effect did not interact with OSPAN, $F(1,26)=$ $.348, p=.560, \eta^{2}=.013$. There was also a main effect of location validity, $\mathrm{F}(1,26)=15.843, p<.01, \eta^{2}=.379$. Replicating Experiments 1 and 2, this location validity effect did not interact with OSPAN, $\mathrm{F}(1,26)=.501, p=.485, \eta^{2}=$ .019. The bottom right of Fig. 3 illustrates the correlation of OSPAN scores with response cuing at validly cued spatial locations, revealing no significant correlation. Also, in replication of Experiments 1 and 2, Fig. 4 illustrates no significant correlation of OSPAN with spatial cuing.

Analysis of group differences is shown in Fig. 3. All groups (except for middle spans at invalid locations) demonstrated significant response cuing effects irrespective of spatial validity. Between-groups comparisons of spatial and response cueing revealed no significant between-group differences at either validly or invalidly cued locations (all $p \mathrm{~s}>.40$ ). All subject groups were able to utilize the combined cues in Experiment 3. 
Table 3 Experiment 3

\begin{tabular}{llll}
\hline & $\begin{array}{l}\text { Valid location } \\
\text { RT (ACC) }\end{array}$ & $\begin{array}{l}\text { Invalid location } \\
\text { RT (ACC) }\end{array}$ & $\begin{array}{l}\text { Spatial cuing } \\
\text { RT (ACC)__ }\end{array}$ \\
\hline $\begin{array}{l}\text { All subjects } \\
\text { Valid orientation }\end{array}$ & $465(.96)$ & $550(.95)$ & $85^{*}(.01)^{*}$ \\
Invalid orientation & $542(.95)$ & $579(.95)$ & $37^{*}(.00)$ \\
Orientation cuing & $77 *(.01)$ & $29 *(.00)$ & \\
High OSPAN & & & $90^{*}(.01)$ \\
Valid orientation & $449(.96)$ & $539(.95)$ & $33(.02)$ \\
Invalid orientation & $530(.96)$ & $24 *(.01)$ & $84^{*}(.01)$ \\
Orientation cuing & $81 *(.00)$ & & $45^{*}(.01)$ \\
Low OSPAN & & $554(.94)$ & \\
Valid orientation & $470(.95)$ & $600(.94)$ & $45^{*}(.00)$ \\
Invalid orientation & $554(.95)$ & & \\
Orientation cuing & $84 *(.00)$ & & \\
\hline
\end{tabular}

\section{Accuracy}

Mean proportion correct for each within-subjects condition was entered into a repeated measures ANOVA with OSPAN as a covariate, $\mathrm{F}(1,26)=.137, p=.714, \eta^{2}=.005$. There were also no significant main effects of either orientation, $\mathrm{F}(1,26)=1.78, p=.193, \eta^{2}=.064$ or location validity, $\mathrm{F}(1,26)=.016, p=.899, \eta^{2}=.001$. Neither effect interacted with OSPAN performance (all $p \mathrm{~s}>.16$ ).

\section{Discussion}

Experiments 1 and 2 were motivated by the hypothesis that the internal generation and representation of feature templates (object files) is the engaging factor in WM performance. We find in Experiment 3 that when these internal representations are not necessary, by dint of changing one sentence of the instructions, variation in WMC has null predictive utility. The important point is that all three experiments used letter cues as the nonspatial cuing stimuli. If the results of Experiments 1 and 2 were driven by differences in the nature of the cues themselves or an interaction between this difference and the ability to maintain task instructions, we would expect at least a weak predictive association between WMC and the orientation cuing found in Experiment 3. Instead, we observe a striking pattern of near equal orientation cuing across all subjects. In fact, in contrast to Experiments 1 and 2, all subject groups demonstrate significant letterdriven cuing effects in Experiment 3. Thus, with greater degree of confidence, we conclude that feature-based attention (when bound to a spatial expectancy) is an engaging factor behind variability in WM performance, and not simply the combination of different types of attentional cues in general.
The results of Experiment 3 also further outline the boundaries of theoretical and experimental associations between the OSPAN task and other cognitive tasks. Kane et al. (2006) note that given the breadth with which this task predicts performance on a number of disparate tasks, a greater degree of discriminate theoretical fidelity is needed. Thus, it is equally important to demonstrate conditions that are not related to our individual variability measure as it is to show a significant relationship. If a task predicts performance in a generalized and non-specific way, it is difficult to use this task to constrain cognitive theory or to rule out general motivational variability among subjects. We find here that WMC is not related to spatial orienting itself or combined cuing where a cue provides information concerning the anticipated correct response.

Experiment 3 also replicates our findings of a null association between WMC and spatial attention across Experiments 1 and 2. Such a finding is consistent with recent studies showing no significant correlation of working memory with covert spatial orienting (Fukuda \& Vogel, 2009) in the absence of distracters. The reason for the present null correlation is a matter of interpretation, however. As with Experiments 1 and 2, it may be a result of insufficient reliability of our measure. However, we find that the lack of correlation in Experiment 3 is also not a function of low reliability with observed split-half reliabilities of $r=.92$ for the spatial cuing effect and $r=.90$ for the orientation cuing effect (Spearman, 1910; Brown, 1910).

An interpretation of our results so far is that spatial and feature-based attentions operate under the restriction of separate pools of attentional resources: that WMC is critically associated with feature-based attention via the shared recruitment of an attentional template. Because space is an inherent property of the environment, no such internal representations are required for the orienting of spatial attention. Furthermore, when we remove the 
component of the task that requires the cue-induced generation of such templates in Experiment 3, variation in WMC has null predictive utility for the orientation cuing task while controlling for other factors. Thus, feature-based attention might tax the same resources necessary for performance on our measure of WMC while spatial attention recruits a fundamentally different pool of resources (as suggested by Courtney, Ungerleider, Keil, \& Haxby, 1996; Hayden \& Gallant, 2005; ) that is not associated with WMC. The demonstrated unique variance shared between feature-based attention and WMC in Experiments 1 and 2 certainly supports this notion. Furthermore, a domain general resource-based account implies that the deployment of spatial attention consumed all of the attentional capacity of the low span subjects and that these subjects do not demonstrate feature-based cuing effects because they have no spare capacity with which to generate a feature-based expectancy. If the resource-based account is true in a general fashion, then it is hard to explain from what pool of resources the low spans drew in order to map the $\mathrm{V}$ and $\mathrm{H}$ cues onto their associated orientation expectancies in Experiment 3 if the deployment of spatial attention taxed their attention pools to the maximum.

Although our results thus far suggest a role of featurebased attention in WM, we have only partly addressed the hypothesis outlined in the introduction. The conclusion at this point could be that feature-based attention itself is associated with WMC in a general fashion. The alternative is that our findings are specific to the cuing paradigm we have devised where an anticipated feature-based expectancy is bound to an anticipated location. It may be that featurebased attention interplays with WMC only when combined with spatial attention. If spatial attention and feature-based attention operate in distinct parallel systems (Courtney et al., 1996), then WM might serve as the workspace upon which these two systems are coordinated. Such a proposition is consistent with recent models of WM and consciousness (Baars \& Franklin, 2003).

\section{Experiment 4}

The degree to which variation in WM is independently associated with feature-based attention and the lack of correlation with spatial attention suggests that these two forms of attention rely on separate sub-systems. Orientation cuing relies on attention to an internal object representation, and this form of attention that might be crucial to performance on the WM task that we employ. An easy interpretation at this point would be to infer that WM and feature-based attention rely on this common attentional system. The demonstrated unique variance between feature based-attention and WMC certainly suggests this. However, it is important to note that the feature-cuing effect observed for high spans in Experiments 1 and 2 only occurred within the spotlight of a cued spatial location. Even though our observed correlation is specific to the orientation cuing effect, our task entails the coordination of spatial and feature-based attention. It may be that high spans excel in the use of the cue to bind two separate forms of attention into an integrated expectancy. The purpose of Experiment 4 is to test whether the observed correlations are specific to integrated spatial and feature-based attentional performance. If integrated attention is the engaging factor driving the shared variance between the observed cuing effects and WMC, then removing the dual aspect of the cuing paradigm and presenting just an orientation cue at fixation to cue upcoming target gratings should produce cuing effects that do not correlate with variation in WMC. If feature-based attention is related to WMC in a general fashion via the shared recruitment of attention to object files, then variation in WMC should co-vary with orientation cuing presented in isolation.

\section{Method}

\section{Participants}

Fifty-two University of California-Davis undergraduates participated for research credit as a requirement for completion of an introductory psychology class. All had normal or corrected-to-normal vision. All trials were entered into statistical analysis, and data from two participants were not included for failure to maintain accuracy of $85 \%$ on the mathematical portion of the OSPAN task.

Experiment 4 was similar to Experiments 1 and 2 except that the dual aspect of the cueing paradigm was removed, and subjects were presented with the letter "V" or and " $\mathrm{H}$ " at fixation and instructed to use this cue to anticipate the upcoming orientation of a grating to be presented at fixation. The target stimuli from Experiment 2 were used, and the cue to target interval was the same as in Experiments 1 and 2. Subjects were instructed to use the cue to anticipate the upcoming target grating and respond as quickly and as accurately as possible to the spatial frequency of each target grating (as in Experiments 1 and 2). Target gratings were presented at fixation (see Fig. 5 for a typical trial). Trials were randomized using a Mersene twister algorithm, and response hands were counterbalanced between subjects. Each participant was presented with 12 blocks of 60 trials each. Each block had 52 validly cued and 8 invalidly cued trials with a total proportion of .85 validly cued trials. Cuing effects are reported as the difference in reaction time between the valid and invalid trials aggregated for each subject. 
Fig. 5 Illustrates an example trial

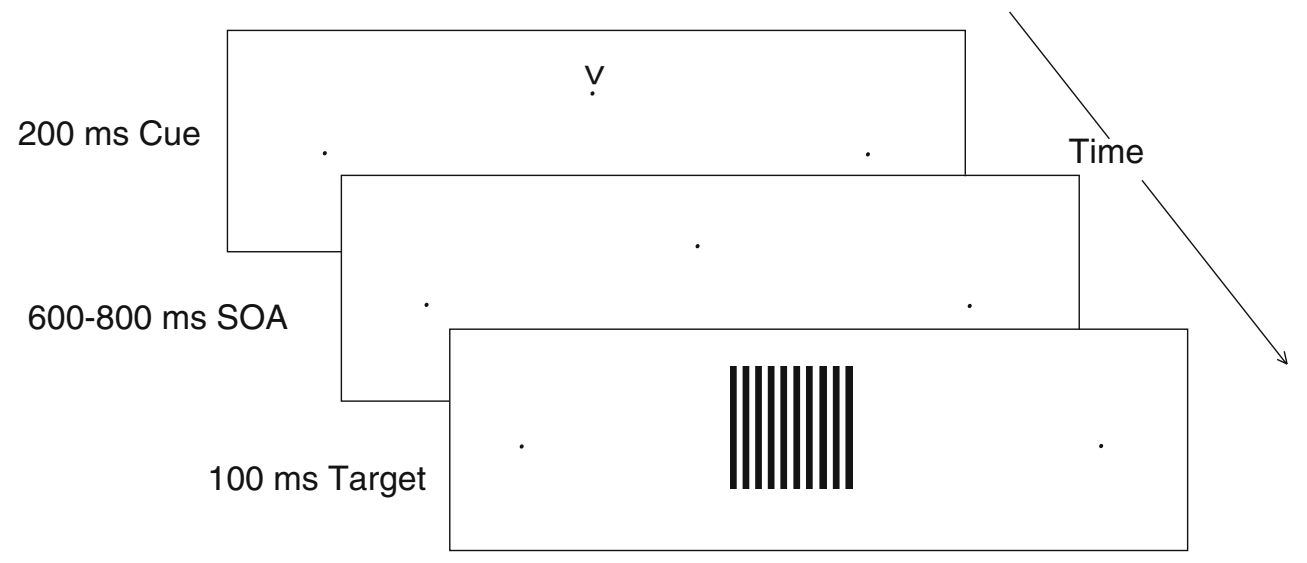

\section{Results}

\section{Reaction times}

Table 4 contains mean reaction times (RTs) in milliseconds and proportion correct for each condition for high and low span groups with significant simple effects marked with an asterisk. Results of a repeated measures ANOVA reveal no significant orientation cueing $\times$ OSPAN interaction, $F(1,48)=$ $.291, p=.596, \eta^{2}=.006$. Analysis of the cuing effect over all subjects revealed a significant main effect of cue validity, $\mathrm{F}(1,48)=30.732, p<.001, \eta^{2}=.390$ showing that participants were utilizing the cues to anticipate the orientation of the upcoming target. Analysis of group differences also revealed no differences between group category (high vs. low tertile split), with no group $\times$ orientation cuing interaction, $\mathrm{F}(1,30)=.047, p=.829$, $\eta^{2}=002$. In fact, both low, $\mathrm{t}(15)=2.880, p=.01$, and high, $t(15)=2.934, p=.01$, spans exhibited significant feature cuing effects. Furthermore, the cuing effect (invalid RTs valid RTs) was not significantly correlated with variation in WMC, $r=.078, p=.592$. Importantly, from Cohen (1977), the power to detect a correlation of .39 (from Experiments 1 and 2) is greater than .80 with an $N=50$ subjects. Reliability of this cuing effect was comparable to the orientation cuing observed in Experiments 1 and 2 at $r=.70$, and betweensubjects variability was sufficient to reveal inter-subject differences with a range of $266 \mathrm{~ms}$ (minimum $=-20 \mathrm{~ms}$; maximum $=246 \mathrm{~ms}$ ) and $\mathrm{a} \mathrm{SD}=63 \mathrm{~ms}$.

\section{Accuracy}

Proportion correct for each condition was entered into a repeated measures ANOVA with OSPAN as a covariate. Results reveal no significant orientation cuing by OSPAN interaction, $\mathrm{F}(1,48)=.783, p=.401, \eta^{2}=.015$. There was a significant effect of cue validity over all subjects however, $\mathrm{F}(1,48)=21.489, p<.001, \eta^{2}=.309$, with higher accuracy for validly cued trials vs. invalidly cued trials. Analysis of group differences also revealed no difference between high and low capacity subjects, $\mathrm{F}(1,48)=.954, p=.337, \eta^{2}=.031$. Also, variation in WMC was not significantly correlated with proportion correct differences between valid and invalidly cued targets, $r=.127, p=.381$.

\section{Discussion}

The results of Experiment 4 suggest that variation in WMC is not a determining factor in the ability of an individual to anticipate the orientation of an upcoming target. We had hypothesized that the shared variance between orientation cuing and WMC in a combined expectancy paradigm might be driven by the shared recruitment of attention to internal representations. In the absence of the dual-cue aspect of Experiments 1 and 2 however, variation in WMC has little predictive utility even though transformation of the orientation cue into an anticipated representation was still required. The lack of predictive utility is not a function of low reliability, low power, or insufficient variability

Table 4 Experiment 4

RT (ACC)

\begin{tabular}{ll}
\hline All subjects & \\
Valid orientation & $637(.82)$ \\
Invalid orientation & $688(.79)$ \\
Orientation cuing & $51^{*}(.03)^{*}$ \\
High OSPAN & \\
Valid orientation & $645(.84)$ \\
Invalid orientation & $694(.81)$ \\
Orientation cuing & $49^{*}(.03)^{*}$ \\
Low OSPAN & \\
Valid orientation & $646(.80)$ \\
Invalid orientation & $690(.79)$ \\
Orientation cuing & $44^{*}(.01)$ \\
\hline
\end{tabular}


between subjects. This null result follows the logic of other studies demonstrating specific circumstances under which $\mathrm{WM}$ is associated with attentional performance.

Other research has shown that spatial orienting (Fukuda \& Vogel, 2009), pro-saccade performance (Kane et al., 2001), and overcoming Stroop interference (Kane \& Engle, 2003 as measured by errors) are only weakly related to variation in WMC. However, each of these studies also demonstrated that when an additional attentional component was added to the task, such as distracter suppression or goal maintenance, variation in WMC predicted performance. Working memory may serve as the workspace under which these systems are coordinated (Baars \& Franklin, 2003). Consistent with this notion is research within the perceptual domain demonstrating that the integration of domain-specific perceptual processes is critically dependent upon attention (Treisman \& Gelade, 1980). In the same way that parallel perceptual systems may be integrated into a percept via attention, parallel attention systems may be coordinated via an integrated workspace that is synonymous with WM. The finding of a critical association between WMC and combined spatial and orientation cuing in Experiments 1 and 2 lends credit to such a proposal. The lack of correlation in Experiment 3 suggests that WMC might specifically interact with attention systems that are anchored within the perceptual system. In experiment 3 , when the generation of an anticipated orientation did not require attention to a perceptually based internal representation (object file), WMC had little predictive utility in the combined cuing paradigm even though spatial attention was simultaneously deployed. This finding coupled with the unique variance between WM and orientation cuing suggests separate percept-based attentional systems that cooperate via WM. Although speculative, the results of Experiments 1 and 2 support the notion that WM serves as a system by which different forms of percept-based attention are integrated. A more immediately derivable conclusion is that variation in WMC predicts orientation cuing when combined with spatial attention, but not response-related expectancies, or orientation cuing in isolation. This finding lends novel converging support for the controlled attention view of WM (Cowan, 2001; Kane et al., 2001).

\section{Conclusions and general discussion}

In summary, these data suggest a specific association of feature-based attention with individual WM performance only when combined with spatial attention. Across Experiments $1-3$, WMC was not correlated with spatial attention itself, but was strongly associated with the ability to generate low-level feature expectancies bound to an anticipated location. Of particular note is that the significant orientation cuing demonstrated by high spans only occurred at validly cued spatial locations, suggesting a hierarchical dependence of feature-based attention upon spatial attention (Mangun, 1995). In striking contrast, no significant feature cueing was observed at invalidly cued spatial locations, nor did performance in this condition interact with WMC. Furthermore, Experiment 3 demonstrated the discriminate validity of this association by demonstrating near equality of performance across all subjects using the same stimuli and an almost identical procedure. Experiment 4 demonstrated that the association between orientation cuing and WMC does not generalize to orientation cuing when presented in isolation. Although our hypothesis was centered on the possible association of WM to featurebased attention, we find that this association is specific to a situation where feature-based attention is combined with spatial attention. Thus, strong claims of an association between feature-based attention and WMC in general are not supported. Instead, this suggests a role for WM in coordinated attentional deployment at the perceptual level (Experiments 1 and 2) but not coordinated attentional deployment in general (Experiment 3). Although the feature-based attention performance in Experiments 1 and 2 was uniquely associated with variation in WMC, it seems this shared variance only occurs when a spatial expectancy is combined with orientation cuing. It is important to note that this type of coordinated attention is a hallmark of a certain type of visual search task and that loading WM interferes when the search task entails this kind of coordinated performance (Woodman et al., 2008). When a search template is consistent across trials, thus not requiring the generation and coordination of a to-be-searched-for template with the deployment of spatial attention throughout the display, loading WM has no effect on search performance (Woodman et al., 2001a, 2001b). Related to this notion is that variation in $\mathrm{WMC}$ is unrelated to the prospective deployment of spatial attention to anticipate target locations in a search display in the absence of distracters (Poole \& Kane, 2009); however, when distracter suppression is added to the visual search task, variation in WMC strongly predicts performance. Our data, along with the data of Fukuda and Vogel (2009), are consistent with these findings in a general fashion. If it is assumed that distracter suppression is an attentional component that is independent of spatial attention, then it appears that variation in WMC is important for integrated attentional performance. Whether feature-based attention and distracter suppression share a common mechanism is a hypothesis that remains to be tested however. Two concerns arise. One is the specific reason for our observed correlation in Experiments 1 and 2. It could still be due to the ability of subjects with higher WMC to excel in the complexity of control of attention 
in general. Such a hypothesis does not fit well with the results of Experiment 3, which, given its dual cue aspect, seems to share equivalent demands on a construct such as complexity of control. Furthermore, a resource-based account suggesting that high spans excel in the amount of information needed to control attention cannot be reconciled with the independent variance shared between WMC and feature-based attention in Experiments 1 and 2, nor does this account seem to be consistent with the findings of Poole and Kane (2009), who demonstrated an equivalent effect of WMC that remained consistent whether 1,2 , or 8 locations were cued. However, more research is needed to see if our findings generalize to the coordination of multiple expectancies within a single perceptual dimension or if WM is specifically associated with integrated attentional performance across distinct perceptual dimensions as our data suggest.

Open Access This article is distributed under the terms of the Creative Commons Attribution Noncommercial License which permits any noncommercial use, distribution, and reproduction in any medium, provided the original author(s) and source are credited.

\section{References}

Anllo-Vento, L., Luck, S. J., \& Hillyard, S. A. (1998). Spatiotemporal dynamics of attention to color: Evidence from human electrophysiology. Human Brain Mapping, 6, 216-238.

Astafiev, S. V., Shulman, G. L., Stanley, C. M., Snyder, A. Z., Van Essen, D. C., \& Corbetta, M. (2003). Functional organization of human intraparietal and frontal cortex for attending, looking, and pointing. The Journal of Neuroscience, 23, 4689-4699.

Awh, E., Jonides, J., \& Reuter-Lorenz. (1998). Rehearsal in spatial working memory. Journal of Experimental Psychology: Human Perception and Performance, 24, 780-790.

Awh, E., \& Vogel, E. K. (2008). The bouncer in the brain. Nature Neuroscience, 11, 5-6.

Baars, B. J., \& Franklin, S. (2003). How conscious experience and working memory interact. Trends in Cognitive Sciences, 7, 166172.

Baddeley, A. (2003). Working memory: Looking back and looking forward. Nature Reviews. Neuroscience, 2003(4), 829-839.

Barnhardt, J., Ritter, W., \& Gomes, H. (2008). Perceptual load affects spatial and nonspatial visual selection processes: An event related potential study. Neuropsychologia, 46, 2071-2078.

Bleckley, M. K., Durso, F. T., Crutchfield, J. M., Engle, \& Khanna, M. M. (2003). Individual differences in working memory capacity predict visual attention allocation. Psychonomic Bulletin \& Review, 10, 884-889.

Brown, W. (1910). Some experimental results in the correlation of mental abilities. British Journal of Psychology, 3, 296-322.

Bundesen, C. (1990). A theory of visual attention. Psychological Review, 97, 523-547.

Cherry, E. C. (1953). Some experiments on the recognition of speech, with one and two ears. The Journal of the Acoustical Society of America, 25, 975-979.

Chiu, Y. C., \& Yantis, S. (2009). A domain-independent source of cognitive control for task sets: Shifting spatial attention and switching categorization rules. The Journal of Neuroscience, 29, 3930-3938.

Cohen, J. (1977). Statistical power analysis for the behavioral sciences. (rev. ed.) Hillsdale, NJ: England: Erlbaum.

Cohen, J., Cohen, P., West, S. G., \& Aiken, L. S. (2003). Applied multiple regression/correlation analysis for the behavioral sciences (3rd ed.). Mahwah: Erlbaum.

Colzato, L. S., Spape, M. A., Pannebakker, M. M., \& Hommel, B. (2007). Working memory and the attentional blink: Blink size is predicted by individual differences in operation span. Psychonomic Bulletin \& Review, 14, 1051-1057.

Conway, A. R. A., Cowan, N., \& Bunting, M. F. (2001). The cocktail party phenomenon revisited: The importance of working memory capacity. Psychonomic Bulletin \& Review, 8, 331-335.

Corbetta, M., Shulman, G. L., Miezin, F. M., \& Petersen, S. E. (1995). Superior parietal cortex activation during spatial attention shifts and visual feature conjunction. Science, 270, 802-805.

Courtney, S. M., Ungerleider, L. G., Keil, K., \& Haxby, J. V. (1996). Object and spatial working memory activate separate neural systems in human cortex. Cerebral Cortex, 6, 39-49.

Cowan, N. (2001). The magical number 4 in short-term memory: A reconsideration of mental storage capacity. The Behavioral and Brain Sciences, 24, 87-114.

Cowan, N. (2005). Working memory capacity limits in a theoretical context. In C. Izawa \& N. Ohta (Eds.), Human learning and memory: Advances in theory and application. The 4th Tsukuba international conference on memory (pp. 155-175). Mahwah: Erlbaum

Desimone, R., \& Duncan, J. (1995). Neural mechanisms of selective visual attention. Annual Review of Neuroscience, 18, 193-222.

Esterman, M., Chiu, Y. C., Tamber-Rosenau, B. J., \& Yantis, S. (2009). Decoding cognitive control in human parietal cortex. Proceedings of the National Academy of Sciences of the United States of America, 106, 17974-17979.

Falk, R. (1998). Replication-A step in the right direction: Commentary on Sohn. Theory and Psychology, 8, 313-321.

Fink, G. R., Dolan, R. J., Halligan, P. W., Marshall, J. C., \& Frith, C. D. (1997). Space-based and object-based visual attention: Shared and specific neural domains. Brain, 129, 2013-2028.

Fisher, R. A. (1966). The design of experiments (8th ed.). Edinburgh: Oliver \& Boyd.

Fukuda, K., \& Vogel, E. K. (2009). Human variation in overriding attentional capture. The Journal of Neuroscience, 29, 87268733.

Handy, T. C., Green, V., Klein, R. M., \& Mangun, G. R. (2001). Combined expectancies: Event-related potentials reveal the early benefits of spatial attention that are obscured by reaction time measures. Journal of Experimental Psychology: Human Perception and Performance, 27, 303-317.

Handy, T. C., \& Mangun, G. R. (2000). Attention and spatial selection: Electrophysiological evidence for modulation by perceptual load. Perception \& Psychophysics, 62, 175-186.

Hayden, B. Y., \& Gallant, J. L. (2005). Time course of attention reveals different mechanisms for spatial and feature-based attention in area V4. Neuron, 47, 637-643.

Hillyard, S. A., \& Anllo-Vento, L. (1998). Event-related potentials in the study of visual selective attention. Proceedings of the National Academy of Science, 95, 781-787.

Hutchison, K. A. (2007). Attentional control and the relatedness proportion effect in semantic priming. Journal of Experimental Psychology. Learning, Memory, and Cognition, 33, 645-662.

Hyun, J. S., Woodman, G. F., Vogel, E. K., Hollingworth, A., \& Luck, S. J. (2009). The comparison of visual working memory representations with perceptual inputs. Journal of Experimental Psychology: Human Perception and Performance, 35, 1140 1160. 
Kane, M. J., Bleckley, M. K., Conway, A. R., \& Engle, R. W. (2001). A controlled-attention view of working-memory capacity. Journal of Experimental Psychology: General, 130, 169-183.

Kane, M. J., \& Engle, R. W. (2003). Working-memory capacity and the control of attention: The contributions of goal neglect, response competition, and task set to Stroop interference. Journal of Experimental Psychology: General, 132, 47-70.

Kane, M. J., Poole, B. J., Tuholski, S. W., \& Engle, R. W. (2006). Working memory capacity and the top-down control of visual search: Exploring the boundaries of "executive attention". Journal of Experimental Psychology: Learning, Memory, and Cognition, 32, 749-777.

Kingstone, A. (1992). Combining expectancies. The Quarterly Journal of Experimental Psychology, 44A, 69-104.

Lavie, N., \& Tsal, Y. (1994). Perceptual load as a major determinant of the locus of selection in visual attention. Perception \& Psychophysics, 56, 184-197.

Long, D. L., \& Prat, C. S. (2002). Working memory and stroop interference: An individual differences investigation. Memory \& Cognition, 30, 294-301.

Luck, S. J., \& Vogel, E. K. (1997). The capacity of visual working memory for features and conjunctions. Nature, 390, 279-281.

Mangun, G. R. (1995). Neural mechanisms of visual selective attention. Psychophysiology, 32, 4-18.

Oh, S. H., \& Kim, M. S. (2004). The role of spatial working memory in visual search efficiency. Psychonomic Bulletin \& Review, 11, 275-281.

Poole, B. J., \& Kane, M. J. (2009) Working-memory capacity predicts the executive control of visual search among distractors: The influences of sustained and selective attention. The Quarterly Journal of Experimental Psychology, 62, 1430-1454.

Posner. (1980). Orienting of attention. The Quarterly Journal of Experimental Psychology, 32, 3-25.

Saults, J. S., \& Cowan, N. (2007). A central capacity limit to the simultaneous storage of visual and auditory arrays in working memory. Journal of Experimental Psychology: General, 136, 663-684.

Shulman, G. L., Ollinger, J. M., Akbudak, E., Conturo, T. E., Snyder, A. Z., Petersen, S. E., \& Corbetta, M. (1999). Areas involved in encoding and applying directional expectations to moving objects. The Journal of Neuroscience, 21, 9480-9496.
Smyth, M. M. (1996). Interference with rehearsal in spatial working memory in the absence of eye movements. The Quarterly Journal of Experimental Psychology, 49, 940-949.

Spearman, C. (1910). Correlation calculated from faulty data. British Journal of Psychology, 3, 271-295.

Treisman, A. M., \& Gelade, G. (1980). A feature-integration theory of attention. Cognitive Psychology, 12, 97-136.

Treisman, A. M., \& Sato, S. (1990). Conjunction search revisited. Journal of Experimental Psychology: Human Perception and Performance, 16, 459-478.

Turner, M., \& Engle, R. (1989). Is working memory task dependent? Journal of Memory and Language, 28, 127-154.

Ungerleider, L. G., \& Haxby, J. V. (1994). "What" and "where" in the human brain. Current Opinion in Neurobiology, 4, 157165.

Unsworth, N., Heitz, R. P., Schrock, J. C., \& Engle, R. W. (2005). An automated version of the operation span task. Behavior Research Methods, 37, 498-505.

Vogel, E. K., \& Awh, E. (2008). How to exploit diversity for scientific gain: Using individual differences to constrain cognitive theory. Current Directions in Psychological Science, 17, 171-176.

Vogel, E. K., McCollough, A. W., \& Machizawa, M. G. (2005). Neural measures reveal individual differences in controlling access to working memory. Nature, 438, 500-503.

Wojciulik, E., \& Kanwisher, N. (1999). The generality of parietal involvement in visual attention. Neuron, 23, 747-764.

Woodman, G. F., \& Luck, S. J. (2004). Visual search is slowed when visuospatial working memory is occupied. Psychonomic Bulletin \& Review, 11, 269-274.

Woodman, G. F., Luck, S. J., \& Schall, J. D. (2008). The role of working memory representations in the control of attention. Cerebral Cortex, 17, 118-124.

Woodman, G. F., Vogel, E. K., \& Luck, S. J. (2001a). Visual search remains efficient when visual working memory is full. Psychological Science, 12, 219-224.

Woodman, G. F., Vogel, E. K., \& Luck, S. J. (2001b). Attention is not unitary. Behavioral and Brain Sciences, 24, 153-154. Comment on Cowan, N. (2001) The magical number 4 in short-term memory: A reconsideration of mental storage capacity. 87-114.

Zhang, W., \& Luck, S. J. (2009). Feature-based attention modulates feed-forward visual processing. Nature Neuroscience, 12, 24-25. 